\title{
VALIDITY OF DIAGNOSTIC COMPUTER-BASED AIR AND FOREHEAD BONE CONDUCTION AUDIOMETRY
}

De Wet Swanepoel ${ }^{1,2}$

Leigh Biagio $^{1}$

1. Department of Communication Pathology, University of Pretoria, South Africa

2. Callier Center for Communication Disorders, School for Behavioral \& Brain Sciences, University of Texas at Dallas, USA

\author{
Corresponding author: \\ De Wet Swanepoel, Ph.D. \\ Associate Professor \\ Department of Communication Pathology \\ University of Pretoria, South Africa, 0002 \\ Tel: +27124202304 \\ Fax: +27 124203517 \\ Email: dewet.swanepoel@up.ac.za
}




\section{ABSTRACT}

Computer-based audiometry allows for novel applications, including remote testing and automation, which may improve the accessibility and efficiency of hearing assessment in various clinical and occupational health settings. This study describes the validity of computer-based diagnostic air and forehead bone conduction audiometry when compared to conventional industry standard audiometry in a soundbooth environment. A sample of 30 subjects (19 to 77 years of age) was assessed with a computer-based (KUDUwave 5000) and industry standard conventional audiometer (GSI 61) to compare air and bone conduction thresholds and test-retest reliability. Air conduction thresholds for the two audiometers corresponded within $5 \mathrm{~dB}$ or less in more than $90 \%$ of instances with an average absolute difference of $3.5 \mathrm{~dB}(3.8 \mathrm{SD})$ and a $95 \%$ confidence interval of 2.6 to $4.5 \mathrm{~dB}$. Bone conduction thresholds for the two audiometers corresponded within $10 \mathrm{~dB}$ or less in $92 \%$ of instances with an average absolute difference of 4.9 $\mathrm{dB}(4.9 \mathrm{SD})$ and a $95 \%$ confidence interval of 3.6 to $6.1 \mathrm{~dB}$. The average absolute test-retest threshold difference for bone conduction on the industry standard audiometer was $5.1 \mathrm{~dB}(5.3$ SD) and for the computer-based audiometer $7.1 \mathrm{~dB}$ (6.4 SD). Computer-based audiometry provided air and bone conduction thresholds within the test-retest reliability limits of industry standard audiometry.

\section{INTRODUCTION}

The development of computer-based audiometry, utilizing a software interface to control testing, allows for a number of novel applications not previously possible with conventional industry standard audiometers. These applications span various areas such as automation of test procedures, telemedicine, integrated data management and real-time quality control features. The 
applications hold significant promise to make audiometric evaluations more accessible and efficient in various clinical and occupational health settings.

An important application for computer-based audiometry is the automation of test procedures. Recent reports have demonstrated that such systems can provide reliable and efficient diagnostic air and bone conduction audiometric evaluations in adults. ${ }^{(1-4)}$ Integrated with a telemedicine approach, automated testing may ensure that diagnostic audiometric services are provided in areas where specialist personnel may be limited or unavailable. ${ }^{(5,6)}$ Results from the automated testing may be uploaded through information and communication technology to be reviewed by specialists in off-site clinics. In some complex or difficult-to-test cases, however, live or synchronous testing may be required. Using desktop-sharing and videoconferencing software computer-based audiometers provide the opportunity for clinicians to test patients remotely through an internet connection. ${ }^{(5,7)}$

Computer-based systems also allow for efficient patient data management. Diagnostic findings may for example be integrated with hearing aid programming software and combined reports may be generated and emailed to other professionals. The diagnostic audiometry software itself may however include quality monitoring indicators. ${ }^{(2,5)}$ For example the response-time of patients can be recorded for each response and if inconsistent or long response times compared to normative ranges are noticed, it may indicate a malingerer or patient requiring reinstruction. A number of such indices, including false-positive and true-positive response rates, may objectively aid the interpretation of test findings..$^{(2,5)}$

A recent computer-based audiometer, the KUDUwave 5000, demonstrated reliable applications for automated audiometry and for remote testing of patients across continents using a synchronous telemedicine setup. ${ }^{(3,7)}$ The audiometer utilizes insert earphones covered by 
circumaural earcups to provide additional attenuation. There are also microphones on the outside and inside of the circumaural earcup to monitor the environmental noise and to determine the amount of attenuation. These features may allow for assessment in sub-optimal environments outside a soundproof booth. Despite the encouraging findings reported for automated audiometry and remote testing with a telemedicine setup the validity of this computer-based system has not been determined compared to the gold standard, namely an industry standard conventional audiometer, in a controlled environment. ${ }^{(3,7)}$ This study therefore investigated the validity of computer-based diagnostic air and forehead bone conduction audiometry using this device when compared to a conventional industry standard audiometer.

\section{PATIENTS AND METHODS}

The project was approved by the institutional ethics committee and all participants were required to provide informed consent before participating. A sample of 30 subjects (age range, 19-77 years; average age, 45 years; 18 women) were recruited and both ears were tested. The vast majority $(82 \%)$ of ears presented with normal pure tone average $(500,1000,2000 \mathrm{~Hz})$ thresholds of $\leq 15 \mathrm{~dB}$ HL. Only $8 \%$ indicated pure tone average $(500,1000,2000 \mathrm{~Hz})$ thresholds of more than $25 \mathrm{~dB}$ HL. Considering thresholds across all frequencies in the sample $(n=420$ thresholds), $22 \%$ were $>15 \mathrm{~dB}$ HL and $10 \%$ were $>25 \mathrm{~dB}$ HL.

\section{Equipment}

A GSI 61 diagnostic Type 1 clinical audiometer was used as the industry standard audiometer. The computer-based audiometer was the KUDUwave 5000 (GeoAxon, South Africa),which is software-controlled and was connected to a Netbook computer (Acer Aspire One PC, Windows XP).Air conduction audiometry was conducted with EAR 3A insert earphones with the Grason-Stadler GSI 61 and with custom insert earphones with the 
KUDUwave using deeply-inserted foam eartips. Bone conduction audiometry was conducted using a Radioear B-71 bone oscillator placed on the forehead with the standard spring headband to measure thresholds using both devices. The spring headband on the KUDUwave is held in place on the center of the circumaural headband by an adjustable fitting. Actual coupling force was not determined for either device. The KUDUwave audiometer uses circumaural earcups, which are placed over the insert earphones to provide additional attenuation for testing in nonoptimal environments. The audiometer hardware is contained within the circumaural earcups which plug into the Netbook via a USB cable. An electronic patient response button is also connected to the device.

Both audiometers were calibrated the day before data collection commenced. Calibration was conducted with a Larson Davis 824 Type 1 sound level meter with G.R.A.S. IEC 711 coupler for insert earphones and an AMC493 Artificial Mastoid on an AEC101 coupler with 2559 1/2" microphone for the Radioear B-71 bone conductor. Insert earphones were calibrated in accordance with ISO 389-2 and the bone oscillator with forehead placement calibrated according to ISO 389-3 ${ }^{(8,9)}$ All tests were conducted within a standard soundproof booth.

\section{Measurements}

All subjects were tested with pure tone air and bone conduction audiometry in both ears with the industry standard and the computer-based audiometers. Pure tone air conduction audiometry was completed across octave-interval frequencies from 125 to $8000 \mathrm{~Hz}$, and at 250 to $4000 \mathrm{~Hz}$ for bone conduction. Forehead bone conduction was conducted with both ears occluded with a deep insertion of the earphone. The occlusion effect is minimized by placing insert earphones down to the bony part of the ear canal, which allows for bone conduction testing with occluded ears. ${ }^{(10,11)}$ All tests were completed on the same day for all subjects. A short rest period 
was provided between tests to ensure subjects were cooperative and attentive. A sub-group of 10 subjects returned within less than a week after the first tests for retest of bone conduction audiometry with each device.

All audiometry tests were conducted by two experienced audiologists. The clinicians were blinded to the results of the previous tests of each participant. Subjects were tested in a counterbalanced manner by ensuring that an equivalent number of subjects were tested by each of the audiometers first and an equivalent number of tests were conducted on each audiometer by the two audiologists. This ensured that test order and the tester did not influence the results.

The test protocols and procedures were identical for audiometry with the two devices. A conventional $10 \mathrm{~dB}$ down and $5 \mathrm{~dB}$ up bracketing method was used to determine air and bone conduction hearing thresholds (Modified Hughson-Westlake method). Testing commenced at $1000 \mathrm{~Hz}$ at $40 \mathrm{~dB} \mathrm{HL}$ and proceeded to higher frequencies. After testing the highest test frequency the lower frequencies were evaluated, starting at $500 \mathrm{~Hz}$ and decreasing to the lower frequencies. If no responses were present at $40 \mathrm{~dB} \mathrm{HL}$ the intensity was increased in steps of 10 $\mathrm{dB}$ until a response was noted.

Whenever the difference between the air conduction thresholds in the test and non-test ear was $75 \mathrm{~dB}$ or more at frequencies $\leq 1000 \mathrm{~Hz}$ and $50 \mathrm{~dB}$ or more at frequencies $>1000 \mathrm{~Hz}$, air conduction thresholds were masked. A masking level of $30 \mathrm{~dB}$ above the air conduction threshold of the non-test ear was used. Bone conduction thresholds were determined with continuous masking in the contralateral ear. A continuous masking level of $20 \mathrm{~dB}$ above the air conduction threshold of the non-test ear was used. ${ }^{(12)}$

\section{Analysis}


Descriptive measures were used to illustrate the correspondence between the air and bone conduction thresholds using the two audiometers. The average differences and the absolute differences between thresholds and their distribution were determined for the two audiometers. Confidence intervals $(95 \%)$ were also calculated. In addition to the agreement between air and bone conduction thresholds between the audiometers, test-retest correspondence was compared for bone conduction thresholds measured with the industry standard and the computer-based audiometers.

\section{RESULTS}

The mean thresholds and standard deviations across the test procedures are included in table I. Average air conduction thresholds were slightly higher at all frequencies for the KUDUwave audiometer except at $8000 \mathrm{~Hz}$. The average threshold difference (industry standard - KUDUwave audiometer) across all frequencies was $-1.4 \mathrm{~dB}(5.0 \mathrm{SD})$. Figure 1 provides a detailed distribution of the correspondence between thresholds for the two audiometers. The KUDUwave air conduction thresholds corresponded within $5 \mathrm{~dB}$ or less of those recorded with the industry standard diagnostic audiometer in $90 \%$ of cases (377/420). The average absolute difference between the air conduction thresholds of the two audiometers (Table II) across all frequencies was $3.5 \mathrm{~dB}(3.8 \mathrm{SD})$ with a $95 \%$ confidence interval of 2.6 to $4.5 \mathrm{~dB}$. The largest difference scores were measured at the lowest and highest frequencies $(125$ and $8000 \mathrm{~Hz})$ with differences of 4.7 and $4.8 \mathrm{~dB}$ respectively. Figure 2 illustrates the threshold correspondence at each frequency.

The mean bone conduction thresholds are illustrated in Table I. The overall average difference was $0.2 \mathrm{~dB}(6.9 \mathrm{SD})$ between the industry standard and KUDUwave audiometers across all frequencies. The distribution of the correspondence between bone conduction 
thresholds for the two audiometers (Figure 1) was $\leq 5 \mathrm{~dB}$ in $75 \%$ of cases, $\leq 10 \mathrm{~dB}$ in $92 \%$ of cases and $\leq 15 \mathrm{~dB}$ in $98 \%$ of cases. Figure 3 illustrates the correspondence of bone conduction thresholds at each frequency. The majority of difference scores that were $10 \mathrm{~dB}$ or more was from the lower frequencies $(250,500,1000 \mathrm{~Hz})$. The average absolute difference between bone conduction thresholds recorded with the two audiometers (Table II) across all frequencies was $4.9 \mathrm{~dB}(4.9 \mathrm{SD})$ with a $95 \%$ confidence interval of 3.6 to $6.1 \mathrm{~dB}$. The largest absolute mean bone conduction difference was at $500 \mathrm{~Hz}(6.1 \pm 4.9 \mathrm{~dB})$ and the smallest was at $4000 \mathrm{~Hz}(3.9 \pm 4.6$ $\mathrm{dB})$.

Table II includes the average test-retest differences for bone conduction audiometry with both audiometers. The average absolute test-retest difference across all bone conduction frequencies ( $\mathrm{n}=300)$ was $5.1 \mathrm{~dB}(5.3 \mathrm{SD})$ for the industry standard audiometer and $7.1 \mathrm{~dB}(6.4$ $\mathrm{SD)}$ for the KUDUwave audiometer. Figure 4 compares the bone conduction threshold correspondence between the two audiometers and within each audiometer (test-retest differences). The correspondence between bone conduction thresholds of the industry standard and the KUDUwave audiometer was similar to the test-retest correspondence of the industry standard audiometer. The test-retest correspondence for the KUDUwave audiometer was poorer, with a $91 \%$ correspondence within $15 \mathrm{~dB}$ compared to a $92 \%$ correspondence within $10 \mathrm{~dB}$ for the industry standard audiometer.

\section{DISCUSSION}

The air conduction thresholds recorded with the computer-based audiometer corresponded with those of the industry standard audiometer within typical air conduction test-retest limits of $5 \mathrm{~dB}$ in more than $90 \%$ of instances. ${ }^{(13,14)}$ The average absolute difference between thresholds recorded with the computer-based and industry standard audiometer $(3.5 \pm 3.8 \mathrm{~dB})$ was virtually 
the same as the absolute test-retest difference $(3.6 \pm 3.9 \mathrm{~dB})$ previously reported for air conduction audiometry across all frequencies as indicated in table II. $^{(3)}$ A recent report also indicated similar average absolute test-retest threshold differences across air conduction frequencies $(4.1 \pm 3.8 \mathrm{~dB})$ as the differences between audiometers in the current study. ${ }^{(1)}$ The $95 \%$ confidence interval for test-retest measurements $(2.3$ to $6.0 \mathrm{~dB})$ in the sample reported by Margolis et al. ${ }^{(1)}$ was also very similar to the difference between thresholds recorded with the industry standard and KUDUwave audiometers (2.6 to $4.5 \mathrm{~dB})$. The air conduction threshold differences between the KUDUwave and industry standard audiometer are therefore well within current test-retest limits.

The bone conduction threshold differences between the industry standard and computerbased audiometers were within typical test-retest variability (10 to $15 \mathrm{~dB}$ ) for bone conduction audiometry. ${ }^{(15)}$ Bone conduction thresholds are prone to increased test-retest variability when compared to air conduction thresholds. ${ }^{(11,13)}$ This is attributed to several factors including the static force applied, location of the bone vibrator, functional state of the middle ear, the position of the lower jaw and distortion of bone vibrators at lower frequencies. ${ }^{(11)}$ The absolute bone conduction threshold difference between the computer-based and industry standard audiometer $(4.9 \pm 4.6 \mathrm{~dB})$ was similar to, and slightly better than, the test-retest difference $(5.8 \pm 5.4 \mathrm{~dB})$ for bone conduction audiometry recently reported by Margolis et al. ${ }^{(1)}$ The $95 \%$ confidence interval for test-retest measurements $(2.9$ to $7.9 \mathrm{~dB})$ reported by Margolis et al. ${ }^{(1)}$ was also very similar to the difference between bone conduction thresholds recorded with the industry standard and KUDUwave audiometers (3.6 to $6.1 \mathrm{~dB})$.

The absolute threshold difference between the two audiometers in the current study was similar to the bone conduction test-retest difference of the industry standard audiometer $(5.1 \pm$ 
$5.3 \mathrm{~dB}$ ). In both the test-retest threshold comparison and the threshold comparison between the two audiometers the bone conduction thresholds were within $10 \mathrm{~dB}$ or less in $92 \%$ of the measurements. The average absolute test-retest difference for bone conduction with the computer-based audiometer $(7.1 \pm 6.4 \mathrm{~dB})$ was slightly higher than that of the industry standard audiometer, particularly at 500, 1000 and $2000 \mathrm{~Hz}$. The differences were, however, still within the sample-based $95 \%$ confidence interval for typical bone conduction test-retest differences $(2.9$ to $7.9 \mathrm{~dB}$ ) reported by Margolis et al. ${ }^{(1)}$ The reason for this slightly larger test-retest difference is unclear. It may in part be due to the bone oscillator joined to the circumaural headset of the computer-based system which results in greater static force variability between placements. ${ }^{(11)}$ The sample size for the test-retest condition was however small and results from a larger sample may be necessary to shed more light on this discrepancy.

There were notable gaps between the average air and bone conduction thresholds recorded with both audiometers at all frequencies except $2000 \mathrm{~Hz}$ (Table I). The largest of these were in the lower frequencies, especially at $250 \mathrm{~Hz}$. Although deep insertion of the insert earphones minimizes the occlusion effect, ${ }^{(10,11)}$ actual placement may not have been deep enough in all subjects to eliminate this effect entirely. There may also be some safety and comfort concerns in certain cases relating to deep insertion of the earphones, especially in the presence of excessive cerumen, which may prohibit deep insertion.

In conclusion, the air and bone conduction thresholds of the computer-based audiometer corresponded to those of the industry standard audiometer within typical test-retest reliability limits. Audiometric testing with a computer-based audiometer with insert earphones, additional circumaural earcup attenuation, and a bone-oscillator assembly therefore provides equivalent thresholds compared to conventional audiometry with an industry standard audiometer - at least 
in a soundbooth test environment. In light of previous reports demonstrating the validity of automation and telemedicine applications using this computer-based audiometer, current findings indicate that it can be used reliably in controlled hearing and occupational healthcare settings. ${ }^{(3,7)}$ Future investigations into the reliability of audiometry in sub-optimal acoustic environments offered by the additional attenuation and monitoring of noise offered by this device is still necessary.

\section{REFERENCES}

1. Margolis R. H., B. R. Glasberg, S. Creeke, and B. C. J. Moore: AMTAS $®$ : Automated method for testing auditory sensitivity: Validation studies. International Journal of Audiology49(3):185-194 (2010).

2. Margolis R. H., G. L. Saly, C. Le, and J. Laurence: Qualind ${ }^{\mathrm{TM}}$ : A method for assessing the accuracy of automated tests. J. Am. Acad. Audiol.18(1):78-89 (2007).

3. Swanepoel de W., S. Mngemane, S. Molemong, H. Mkwanazi, and S. Tutshini: Hearing assessment-reliability, accuracy, and efficiency of automated audiometry. Telemed. J. E. Health.16(5):557-563 (2010).

4. Ho A. T. P., A. J. Hildreth, and L. Lindsey: Computer-assisted audiometry versus manual audiometry. Otology and Neurotology30(7):876-883 (2009).

\section{Swanepoel D. W., J. L. Clark, D. Koekemoer, J. W. Hall Iii, M. Krumm, D. V. Ferrari, et al:}

Telehealth in audiology: The need and potential to reach underserved communities. International Journal of Audiology49(3):195-202 (2010).

6. Swanepoel W., B. O. Olusanya, and M. Mars: Hearing health-care delivery in sub-Saharan Africa - a role for tele-audiology. J. Telemed. Telecare16(2):53-56 (2010). 
7. de Swanepoel W., D. Koekemoer, and J. Clark: Intercontinental hearing assessment - a study in teleaudiology. J. Telemed. Telecare16(5):248-252 (2010).

8. ISO 389-2: Acoustics -- Reference zero for the calibration of audiometric equipment -- Part 2:

Reference equivalent threshold sound pressure levels for pure tones and insert earphones. International Organization for Standardization (1994).

9. ISO 389-3: Acoustics - Reference zero for the calibration of audiometric equipment Part 3: Reference equivalent threshold force levels for pure tones and bone vibrators. International Organization for Standardization (1994).

10. Dean M. S. and F. N. Martin: Insert earphone depth and the occlusion effect. Am. J. Audiol.9(2):131-134 (2000).

11. Stenfelt S. and R. L. Goode: Bone-conducted sound: Physiological and clinical aspects. Otology and Neurotology26(6):1245-1261 (2005).

12. American Speech-Language-Hearing Association: Guidelines for Manual Pure-Tone Threshold Audiometry [Guidelines]. (2005).

13. Stuart A., R. Stenstrom, C. Tompkins, and S. Vandenhoff: Test-retest variability in audiometric threshold with supraaural and insert earphones among children and adults. Audiology30(2):82-90 (1991).

14. Smith-Olinde L., N. Nicholson, C. Chivers, P. Highley, and D. K. Williams: Test-retest reliability of in situ unaided thresholds in adults. Am. J. Audiol.15(1):75-80 (2006).

15. Roeser R. J. and J. L. Clark: Pure-Tone Tests. In Audiology Diagnosis, R. J. Roeser, M. Valente, and H. Hosford-Dunn (eds.), pp. 238-260. New york: Thieme, 2007. 


\section{FIGURES}

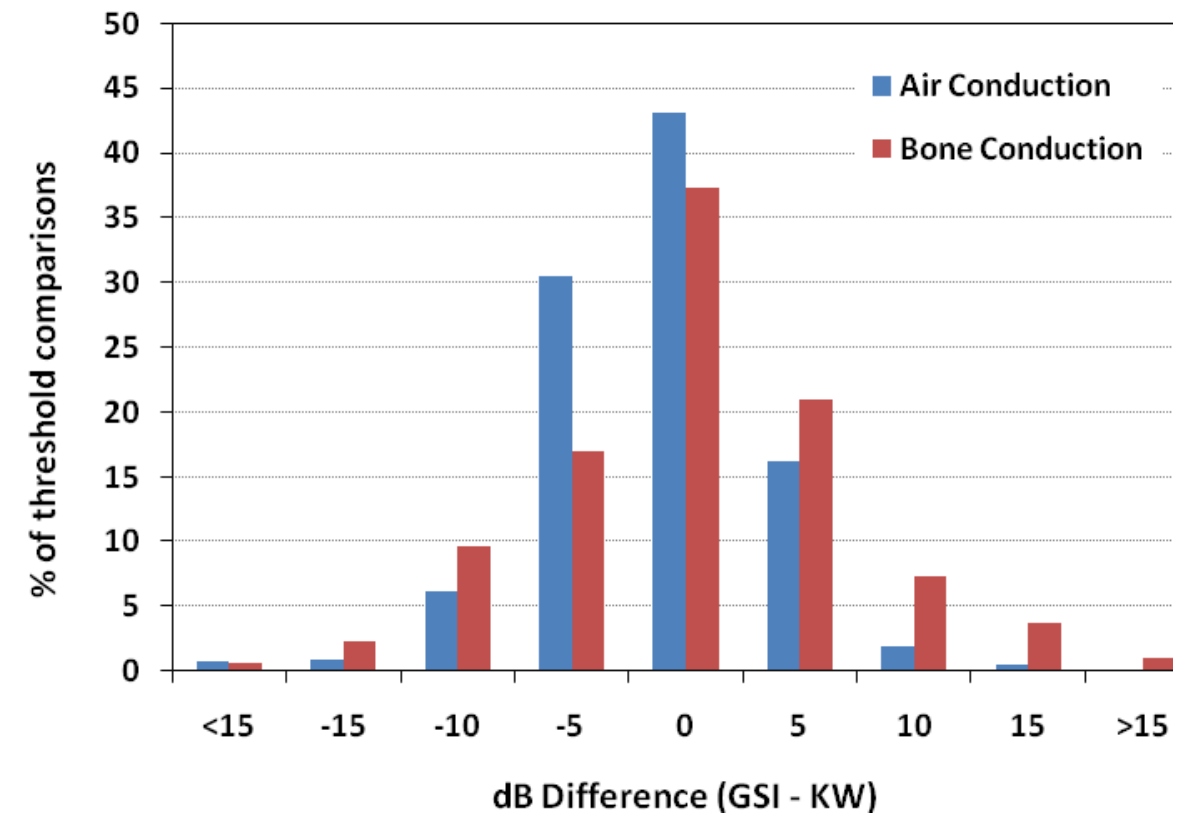

FIGURE 1. Difference between all corresponding GSI and KUDUwave air conduction thresholds $(n=420)$ and bone conduction thresholds $(n=300)$

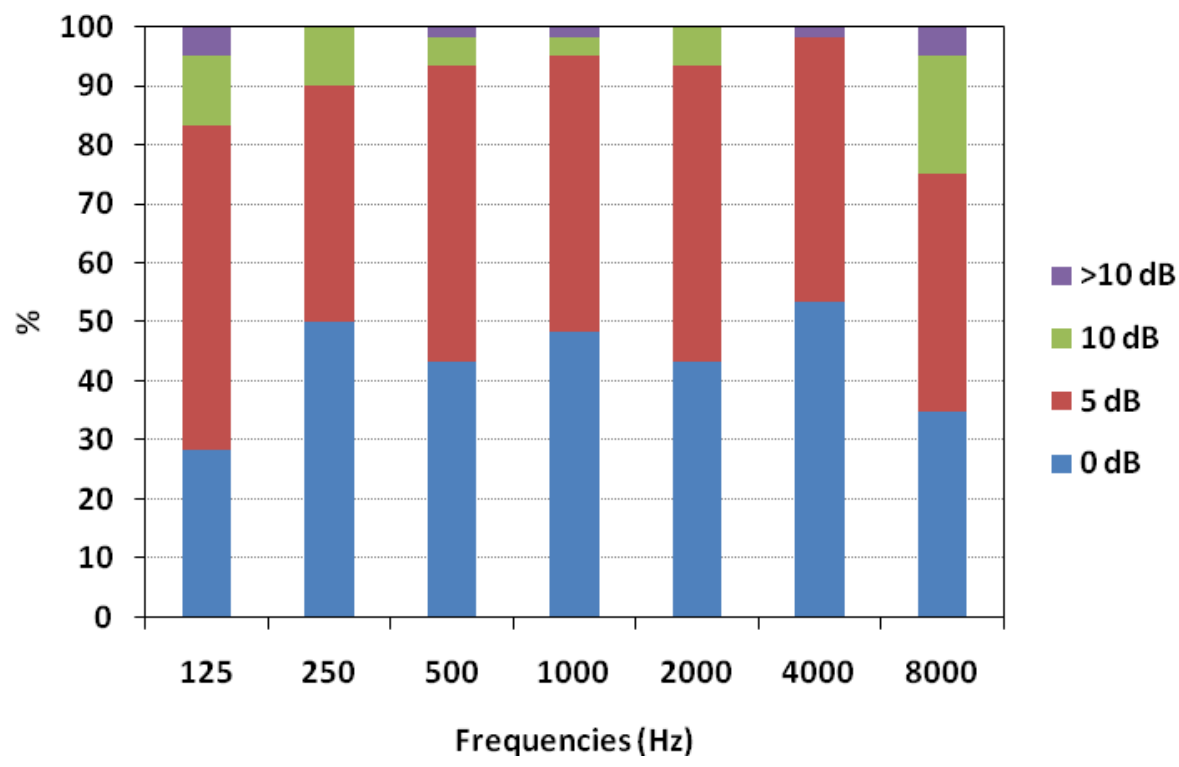


FIGURE 2. Distribution of absolute differences between corresponding air conduction thresholds recorded with the KUDUwave and industry standard audiometer.

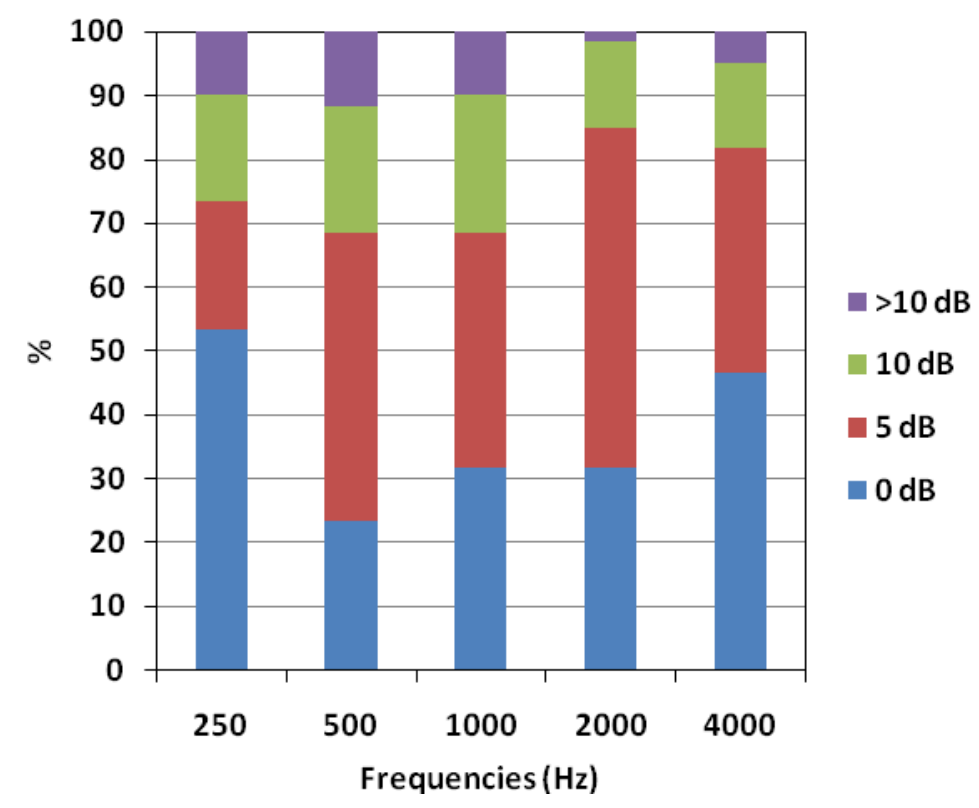

FIGURE 3. Distribution of absolute differences between corresponding bone conduction thresholds recorded with the KUDUwave and industry standard audiometer.

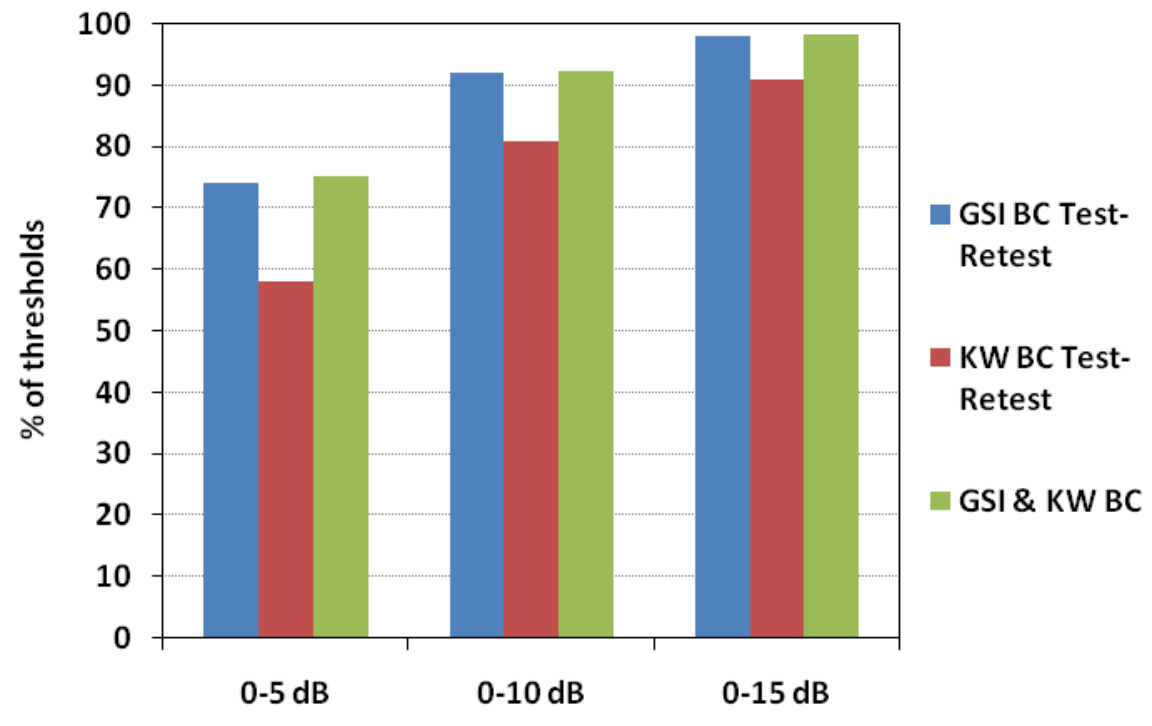

Range of threshold correspondence 
FIGURE 4. Bone conduction threshold correspondence between the (1) industry standard audiometer (GSI) and the KUDUwave audiometer (KW) on 30 subjects ( $n=60$ ears); the testretest threshold correspondence on a sub-group of 10 subjects ( $n=20$ ears) of (2) the industry standard audiometer (GSI) and; (3) the KUDUwave audiometer (KW).

\section{TABLES}

TABLE I. Mean air conduction (AC) and bone conduction (BC) thresholds (dB HL) for the industry standard (GSI) and KUDUwave (KW) audiometers ( $\mathrm{n}=60$ ears).

\begin{tabular}{ccccccccc}
\hline \multicolumn{7}{c}{ Frequencies (Hz) } \\
\hline & $\mathbf{1 2 5}$ & $\mathbf{2 5 0}$ & $\mathbf{5 0 0}$ & $\mathbf{1 0 0 0}$ & $\mathbf{2 0 0 0}$ & $\mathbf{4 0 0 0}$ & $\mathbf{8 0 0 0}$ & ALL \\
\hline AC GSI & 6.9 & 6.2 & 7.6 & 9.2 & 9.6 & 13.1 & 21.0 & $\mathbf{1 0 . 5}$ \\
(SD) & $(9.5)$ & $(9.4)$ & $(10.4)$ & $(10.8)$ & $(13.2)$ & $(17.7)$ & $(22.5)$ & $\mathbf{( 1 4 . 8 )}$ \\
AC KW & 10.6 & 7.8 & 9.3 & 10.3 & 10.9 & 13.3 & 20.9 & $\mathbf{1 1 . 9}$ \\
(SD) & $(10.0)$ & $(9.8)$ & $(11.0)$ & $(11.2)$ & $(13.6)$ & $(17.9)$ & $(21.7)$ & $\mathbf{( 1 4 . 7 )}$ \\
BC GSI & & -5.8 & 3.7 & 2.7 & 10.8 & 7.2 & & $\mathbf{3 . 8}$ \\
(SD) & & $(6.3)$ & $(10.5)$ & $(11.1)$ & $(14.7)$ & $(16.2)$ & & $\mathbf{( 1 3 . 4 )}$ \\
BC KW & & -7.1 & 2.9 & 4.2 & 10.7 & 6.8 & & $\mathbf{3 . 5}$ \\
(SD) & & $(5.0)$ & $(10.4)$ & $(10.6)$ & $(13.8)$ & $(16.0)$ & & $\mathbf{( 1 3 . 1 )}$ \\
\hline
\end{tabular}

TABLE II. Average absolute threshold differences (\&standard deviation). Comparison between the GSI and $\mathrm{KW}$ thresholds (air and bone conduction) and test-retest threshold differences. $\mathrm{AC}=$ air conduction; $\mathrm{BC}=$ bone conduction; $\mathrm{GSI}=$ GrasonStadler audiometer; $\mathrm{KW}=$ KUDUwave audiometer.

\begin{tabular}{|c|c|c|c|c|c|c|c|c|}
\hline \multicolumn{9}{|c|}{ Frequencies (Hz) } \\
\hline $\begin{array}{c}\text { dB } \\
\text { Difference }\end{array}$ & 125 & 250 & 500 & 1000 & 2000 & 4000 & 8000 & $A L L$ \\
\hline AC (GSI-KW) & $4.7(3.9)$ & $3.0(3.3)$ & $3.5(4.5)$ & $3.0(3.6)$ & $3.2(3.0)$ & $2.6(3.4)$ & $4.8(4.4)$ & $3.5(3.8)$ \\
\hline$\underset{\text { retest }^{\mathrm{A}}}{\text { AC KW Test- }}$ & $4.8(5.0)$ & $3.8(3.7)$ & $3.3(3.7)$ & $3.7(3.4)$ & $3.0(3.6)$ & $3.0(3.5)$ & $3.3(4.3)$ & $3.6(3.9)$ \\
\hline $\begin{array}{c}\text { BC (GSI - } \\
\text { KW) }\end{array}$ & & $4.3(5.6)$ & $6.1(4.9)$ & $5.7(5.3)$ & $4.3(3.5)$ & $3.9(4.6)$ & & $4.9(4.9)$ \\
\hline $\begin{array}{c}\text { retest }^{\text {BC GSI }} \\
\text { re }\end{array}$ & & $4.0(5.5)$ & $5.3(4.4)$ & $5.5(7.4)$ & $3.8(3.6)$ & $7.0(4.7)$ & & $5.1(5.3)$ \\
\hline $\begin{array}{c}\text { BC KW Test- } \\
\text { retest }^{\mathrm{C}}\end{array}$ & & $3.5(5.2)$ & $10.5(5.4)$ & $8.0(7.5)$ & $7.8(6.0)$ & $5.8(6.1)$ & & $7.1(6.4)$ \\
\hline
\end{tabular}

${ }^{\mathrm{A}}$ Test-retest absolute differences previously reported for KUDUwave AC thresholds by Swanepoel et al. ${ }^{(3)}$

${ }^{B} \&$ C Sub-group used to determine test-retest reliability for bone conduction constituting 10 subjects (20 ears) 